\title{
How Globally Aromatic Are Six-Porphyrin Nanorings?**
}

Jie-Ren Deng, ${ }^{[a] \dagger}$ David Bradley, ${ }^{[b] \dagger}$ Michael Jirásek, ${ }^{[c]}$ Harry L. Anderson, ${ }^{*[a]}$ and Martin D. Peeks*[b]

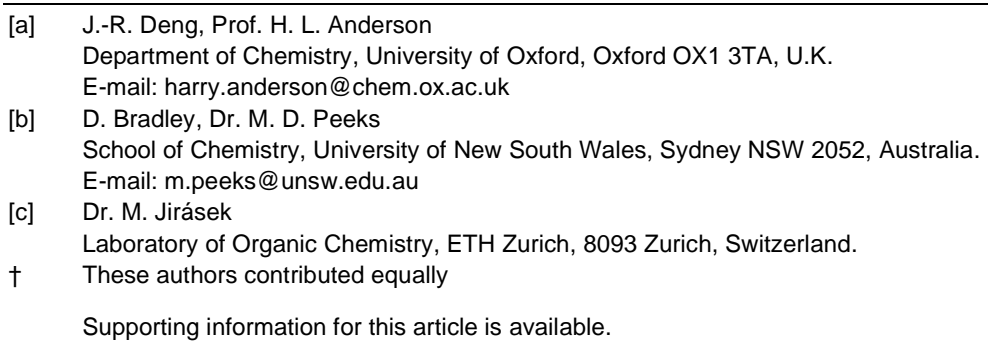

\begin{abstract}
A recent Research Article published in this journal by Matito and coworkers claimed that none of the oxidation states of a butadiyne-linked six-porphyrin nanoring exhibit global aromaticity or antiaromaticity. Here we show that this conclusion is incorrect. A combination of density functional theory (DFT) calculations and experimental NMR data provides compelling evidence for global (anti)aromaticity in a variety of six-porphyrin nanorings in their $2+, 4+$ and $6+$ oxidation states. The strength of the predicted ring current depends on the choice of DFT functional, so it is crucial to use a functional that reproduces the experimental ${ }^{1} \mathrm{H}$ NMR chemical shifts in these cations.
\end{abstract}

The concept of aromaticity is fundamental to many areas of chemistry, and various criteria have been developed for deciding whether a molecule is aromatic. ${ }^{[1]}$ These criteria are not always in agreement, which can lead to controversy. Such disagreements are valuable opportunities to refine our understanding of aromaticity, and resolving them enables the field to move forward. Recently, we published experimental evidence for global aromaticity and antiaromaticity in porphyrinbased nanorings with Hückel circuits of up to $162 \pi$-electrons. ${ }^{[2-8]}$ These macrocycles are dramatically larger than previouslyreported aromatic rings, and we have suggested that studies on such large macrocycles could shed light on the similarity between aromatic ring currents and persistent currents in nonmolecular quantum rings. ${ }^{[8]}$ They may also help elucidate a link between aromatic ring currents and the currents that flow through molecular wires driven by a voltage bias. We analyzed the NMR spectra of porphyrin nanorings (c-PM[bxe $\mathbf{b}_{\mathbf{y}}$, Figure 1) in a range of oxidation states $(Q=-6$ to +12$)$ and concluded that the ${ }^{1} \mathrm{H}$ NMR spectra reveal the presence of global aromatic and antiaromatic ring currents in many of these species. ${ }^{[2-3,5,7.8]}$ Whenever a global ring current was detected, it had the direction predicted by Hückel's rule (i.e. diatropic/aromatic for $4 n+2 \pi$ electrons and paratropic/antiaromatic for $4 n$ m-electrons). ${ }^{13} \mathrm{C}$ NMR and ${ }^{19} \mathrm{~F}$ NMR (for $\boldsymbol{c}-\mathbf{P} 6\left[\mathbf{b}_{6}\right] \cdot \mathbf{T} \mathbf{F}_{\mathrm{F}}{ }^{Q}$ ) chemical shifts, where measured, support the conclusions from ${ }^{1} \mathrm{H}$ NMR spectra. ${ }^{[5]}$ Global (anti)aromaticity was also confirmed by magnetic susceptibility measurements, ${ }^{[2]}$ and by analysis of oxidation potentials and rates of conformational exchange. ${ }^{[7]}$ Global ring currents were not detected in the ground states of neutral nanorings, but variation in the fluorescence behavior of neutral $c-P M\left[b_{N}\right]$ cyclic oligomers as a function of ring size, $N$, indicate that the neutral singlet excited states exhibit global (anti)aromaticity. ${ }^{[4]}$ (a)

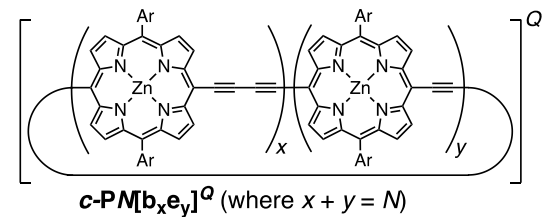

(b)
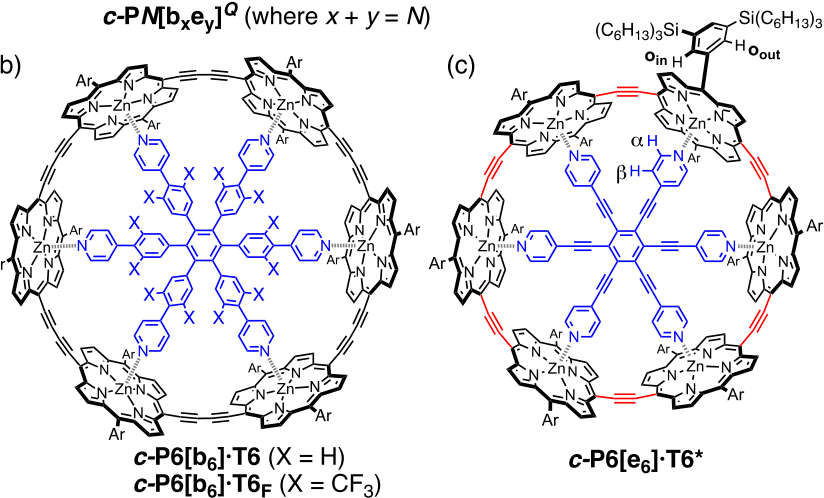

(d)
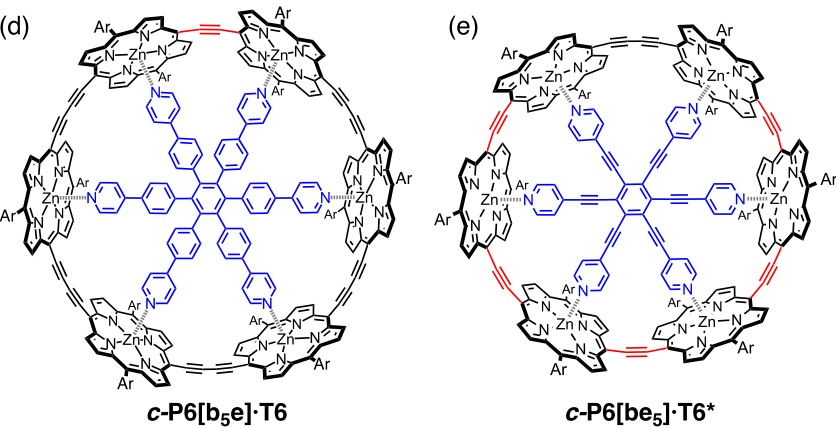

Figure 1. Structures of porphyrin nanorings. (a) General structure for the family of nanorings; $Q$ is the charge (oxidation state); $N, x$ and $y$ are the numbers of porphyrins, butadiynes and ethyne links, respectively. (b)-(e) Structures of six-porphyrin nanoring template complexes. The key ${ }^{1} \mathrm{H}$ NMR resonances, $O_{\text {in }}, O_{\text {out }}, \alpha$ and $\beta$ defined in (c) apply to all the nanoring complexes. All Ar groups are 3,5-bis(trihexylsilyl)phenyl.

In a recent Research Article, ${ }^{[9]}$ Casademont-Reig, GuerreroAvilés, Ramos-Cordoba, Torrent-Sucarrat, and Matito compared our experimental ${ }^{1} \mathrm{H}$ NMR chemical shifts for $\boldsymbol{c}-\mathrm{P} 6\left[\mathbf{b}_{6}\right]^{Q}(Q=0$, $+4,+6$ and +12 ) with those that they calculated using two different functionals, B3LYP and CAM-B3LYP. The chemical shifts calculated by CAM-B3LYP are closer to the experimental values, and this functional does not predict a global ring current in any of the oxidation states. This result led Matito and coworkers to conclude that the cations are not globally (anti)aromatic. Here we show that other density functional approximations (DFAs, often simply referred to as "functionals") 
predict ${ }^{1} \mathrm{H}$ NMR chemical shifts that match experimental values better than either B3LYP and CAM-B3LYP, and that these other DFAs predict global aromaticity in $c-P 6\left[b_{6}\right]^{Q}$, when $Q=+2$ or +6 , and global antiaromaticity when $Q=+4$. Furthermore, the pattern of experimental and computational results for the family of six-porphyrin nanoring cations, $c-\mathbf{P} 6\left[\mathbf{b}_{6}\right] \cdot \mathbf{T 6}^{Q}, c-\mathrm{P} 6\left[\mathbf{e}_{6}\right] \cdot \mathbf{T 6}^{\star}{ }^{Q}$,

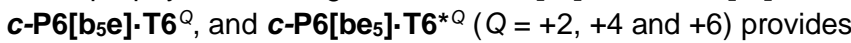
unambiguous evidence for global (anti)aromatic ring currents.

The choice of DFA is critical to the result of DFT calculations and it is well known that hybrid DFAs with a low proportion of exact (Hartree-Fock) exchange, like B3LYP, tend to exaggerate the delocalization of electrons owing to the self-interaction error in DFT. ${ }^{[10]}$ Range-separated functionals like CAM-B3LYP and LC- $\omega$ hPBE vary the amount of exact exchange as a function of distance and can substantially correct this problem. But there is currently no uniquely perfect DFA (indeed, it would probably not be an "approximation" if there were), and the applicability of a given DFA continues to prove system-specific. Here we compare the ability of various DFAs to predict the ${ }^{1} \mathrm{H}$ NMR chemical shift in $c$-P6 $\left[b_{6}\right] \cdot \mathbf{T 6}^{Q}$ for $Q=0,+4,+6$ and +12 . We focus on the three resonances which provide the best probes for magnetic shielding from global (anti)aromaticity, and which are thus most closely linked to observable of interest: the difference in chemical shift between the inner and outer ortho-aryl proton, $\Delta \delta(o)=\delta\left(o_{\text {in }}\right)-\delta\left(o_{\text {out }}\right)$; the difference in chemical shift between $\alpha$-proton of the template complex and that of the free unbound template, $\Delta \delta(\alpha)=\delta(\alpha)-\delta\left(\alpha_{\text {free }}\right)$; and similarly $\Delta \delta(\beta)=\delta(\beta)-$ $\delta\left(\beta_{\text {free }}\right)$ (see Figure $1 \mathrm{c}$ for atom labels). The calculated and experimental shifts are compared in Table 1. When Matito and coworkers carried out this type of analysis, they focused on just two DFAs: B3LYP and CAM-B3LYP, and they concluded that the experimental shifts match most closely with predictions from CAM-B3LYP. The data in Table 1 show that two other DFAs, LC- $\omega$ hPBE $(\omega=0.1)$ and BLYP35, match the experimental data better than either CAM-B3LYP or B3LYP. The same result (i.e. that BLYP35 provides the best match to experiment) is also found when we compare all of the experimental ${ }^{1} \mathrm{H}$ NMR resonances (SI Table S3). When considering the data in Table 1 , we focus on the $Q=+4$ and +6 oxidation states, because these are the systems with indications of global antiaromaticity and aromaticity, whereas the $Q=0$ and +12 states have local rather than global ring currents. In $\boldsymbol{c}-\mathbf{P} 6\left[\mathbf{b}_{6}\right] \cdot \mathbf{T 6}^{4+}$, CAM-B3LYP completely fails to reproduce the sign or magnitude of the shifts $\Delta \delta(\alpha)$ and $\Delta \delta(\beta)$, while B3LYP grossly exaggerates these shifts; only BLYP35 predicts shifts that are close to the experimental values. Similarly with $\boldsymbol{c}-\mathbf{P} \mathbf{6}\left[\mathbf{b}_{6}\right] \cdot \mathbf{T 6}^{6+}$, BLYP35 reproduces the experimental chemical shifts better than CAM-B3LYP or B3LYP. The emerging picture is that whereas B3LYP over-estimates the extent of (anti)aromaticity, CAM-B3LYP goes too far the other way, and fails to reproduce the salient features of the experimental NMR spectra. The success of BLYP35 in accurately describing the behavior of partially oxidized nanorings is perhaps not surprising, because this DFA was originally developed to model organic mixed-valence systems. ${ }^{[11]}$
Table 1. Selected ${ }^{1} \mathrm{H}-\mathrm{NMR}$ chemical shift differences for $\boldsymbol{c}-\mathbf{P} 6\left[\mathbf{b}_{6}\right] \cdot \mathbf{T 6}^{Q}$ (in ppm).

\begin{tabular}{|c|c|c|c|c|c|}
\hline$Q$ & method & $\Delta \delta(0)$ & $\Delta \delta(\alpha)$ & $\Delta \delta(\beta)$ & $\mathrm{RMSE}^{[\mathrm{a}]}$ \\
\hline \multirow[t]{5}{*}{0} & experimental & 0.26 & -6.31 & -2.34 & - \\
\hline & CAM-B3LYP $[\mathrm{b}]$ & 0.09 & -6.34 & -2.54 & 0.15 \\
\hline & B3LYP[b] & 0.31 & -5.34 & -1.97 & 0.60 \\
\hline & LC- $\omega$ hPBE $(\omega=0.1)$ & 0.39 & -5.24 & -2.00 & 0.65 \\
\hline & BLYP35 & 0.44 & -5.79 & -2.31 & 0.32 \\
\hline \multirow[t]{5}{*}{+4} & experimental & - & 14.16 & 12.57 & - \\
\hline & CAM-B3LYP[b] & 0.41 & -1.92 & -0.44 & 14.6 \\
\hline & B3LYP $[\mathrm{b}]$ & 36.36 & 60.43 & 49.10 & 41.7 \\
\hline & LC- $\omega$ hPBE $(\omega=0.1)$ & 4.27 & 4.80 & 4.96 & 8.53 \\
\hline & BLYP35 & 8.56 & 12.08 & 10.80 & 1.93 \\
\hline \multirow[t]{5}{*}{+6} & experimental & -1.87 & -2.83 & -2.06 & - \\
\hline & CAM-B3LYP[b] & -0.19 & -0.97 & -0.36 & 1.74 \\
\hline & B3LYP ${ }^{[b]}$ & -7.23 & -12.26 & -9.85 & 7.71 \\
\hline & LC- $\omega$ hPBE $(\omega=0.1)$ & -1.03 & -2.53 & -1.54 & 0.60 \\
\hline & BLYP35 & -1.17 & -1.99 & -1.37 & 0.75 \\
\hline \multirow[t]{5}{*}{+12} & experimental & -0.74 & 2.77 & 1.50 & - \\
\hline & CAM-B3LYP[b] & -0.91 & 1.88 & 1.13 & 0.57 \\
\hline & B3LYP ${ }^{[b]}$ & -2.67 & 5.63 & 2.68 & 2.10 \\
\hline & LC- $\omega$ hPBE $(\omega=0.1)$ & -2.76 & 5.33 & 2.67 & 2.00 \\
\hline & BLYP35 & -1.53 & 3.09 & 1.64 & 0.50 \\
\hline
\end{tabular}

[a] The root-mean square error (RMSE) values are calculated from the $\Delta \delta$ shown in this table and are with respect to experiment. [b] Data from Matito and coworkers, ref [9]

Matito and co-workers noted that the geometry found by B3LYP was significantly different to those calculated using CAMB3LYP, M06-2X, and LC- $\omega$ hPBE $(\omega=0.2)$. Unfortunately, experimental geometries are not available for any of the oxidized nanorings. Matito and coworkers used single-point calculations at the DLPNO-CCSD(T) (domain localized pair natural orbital, coupled-cluster singles doubles and perturbative triplets) level to compare the energies of calculated geometries from different DFAs. Even with this method's excellent scaling behavior, only relatively modest basis sets are affordable for the nanorings (Matito and coworkers used def-SVP/C). Recent studies have suggested that DLPNO-CCSD $(\mathrm{T})$ may struggle to describe systems with unusual electronic delocalization, such as some Möbius expanded porphyrins. ${ }^{[12]}$ We argue that the DFA is best selected by comparison to an experimental observable that is relevant to the problem at hand - for this problem those data are NMR chemical shifts, and we are fortunate to have a wealth of experimental results on this class of compounds.

Having shown that the experimental ${ }^{1} \mathrm{H}$ NMR data are modeled well by BLYP35, the next question to consider is whether this DFA predicts global antiaromaticity in $c-\mathbf{P} 6\left[\mathbf{b}_{6}\right] \cdot \mathbf{T 6}^{\mathbf{4}^{+}}$ and aromaticity in $\mathbf{c}-\mathbf{P} 6\left[\mathbf{b}_{6}\right] \cdot \mathbf{T 6}^{6+}$. A popular way to answer this 
question is to compute a grid of nuclear magnetic shielding in space around the molecule, known as a NICS grid. The plots of $\operatorname{NICS}(0)_{\text {iso }}$ and $\operatorname{NICS}(0)_{z z}$ in Figure 2 indicate there is indeed a global paratropic ring current in the +4 state and a global diatropic current in the +6 state. The same conclusion comes from NICS plots calculated using LC- $\omega$ hPBE $(\omega=0.1$, SI Figure S4), which also fits the experimental data better than either CAM-B3LYP or B3LYP. ${ }^{[5,8]}$ In the discussion above, we have focused on a few ${ }^{1} \mathrm{H}$ NMR resonances, but the conclusions are also supported by experimental data from other ${ }^{1} \mathrm{H}$ signals, such as those on the trihexylsilyl chains, or by analyzing ${ }^{13} \mathrm{C} N M R$ signals and ${ }^{19} \mathrm{~F}$ NMR resonances in $c-P 6\left[b_{6}\right] \cdot \mathbf{T 6}_{\mathrm{F}}{ }{ }^{[5]}$

In our second experimental study of nanoring cation global aromaticity, we reported the ${ }^{1} \mathrm{H}$ NMR spectrum of the dication, $c$ P6 $\left[\mathbf{b}_{6}\right]^{2+}{ }^{[5]}$ Consistent with Hückel's rules, this $82 \pi$-electron species is aromatic according to the magnetic criterion, based on both experimental NMR spectroscopy and previouslyreported calculations with LC- $\omega$ hPBE $(\omega=0.1) .{ }^{[5]}$ For low values of $Q / N$, one might expect a degree of open-shell character. We found that when we treated $c-\mathbf{P} 6\left[\mathbf{b}_{6}\right] \cdot \mathbf{T 6}^{2+}$ in the same way as the other oxidation states, as a closed-shell singlet, BLYP35 dramatically over-stated the aromaticity (SI Figure S8). On the other hand, the triplet state resulted in antiaromaticity, consistent with Baird's rule but contrary to experiment. A broken-symmetry unrestricted singlet-state calculation resulted in NMR chemical shift differences in relatively close agreement to experiment (SI Table S12). While this result is useful for connecting experimental observables to computational metrics of aromaticity (e.g. the NICS in Figure 2), we emphasize that our computational study of the dication is limited in scope and completeness, owing to the huge size of the system. Furthermore, the spin state and temperature-dependence of this system has not yet been explored experimentally. The picture is clearer for the +4 and +6 states: calculations with the BLYP35 and LC- $\omega$ hPBE functionals reproduce our experimental results and give clear insight into the global antiaromaticity and aromaticity of these charged nanorings.

We next turn to the question posed in the title of Matito and coworkers' article: "How aromatic are molecular nanorings?" Although there is no generally accepted method for quantifying aromaticity, one approach is to estimate the ring current susceptibility (in $n A / T$ ), i.e. the magnitude of the ring current (in $n A)$ that would be induced by an external field of $1 T^{[8,13]}$ (sometimes called 'ring current strength' ${ }^{[14]}$ ). If we can identify a chemical shift difference, $\Delta \delta$, that is attributable to a ring current effect, then we can estimate the magnitude of that ring current using equation (1) ${ }^{[8]}$

$$
\Delta \delta=(/ / B) \text { RCGF }
$$

where $I$ is the current, $B$ is the external magnetic field, $I / B$ is the ring current susceptibility and RCGF is the ring current geometric factor, which is calculated from the molecular geometry using the Biot-Savart law, and which quantifies the sensitivity of a given point in space to the induced magnetic field of the ring current. ${ }^{[15]}$ The consistency between ring current susceptibilities from experimental shifts and those calculated using the BLYP35 functional is illustrated by the plot in Figure 3a We adopt the convention that diatropic ring currents have negative sign, and paratropic positive. The ring current susceptibilities are consistent with the presence of global aromaticity and antiaromaticity in the $Q=2,4$ and 6 oxidation states of $c-\mathbf{P} 6\left[\mathbf{b}_{6}\right] \cdot \mathbf{T} \mathbf{6}^{Q_{+}}$, in accord with Hückel's rules.

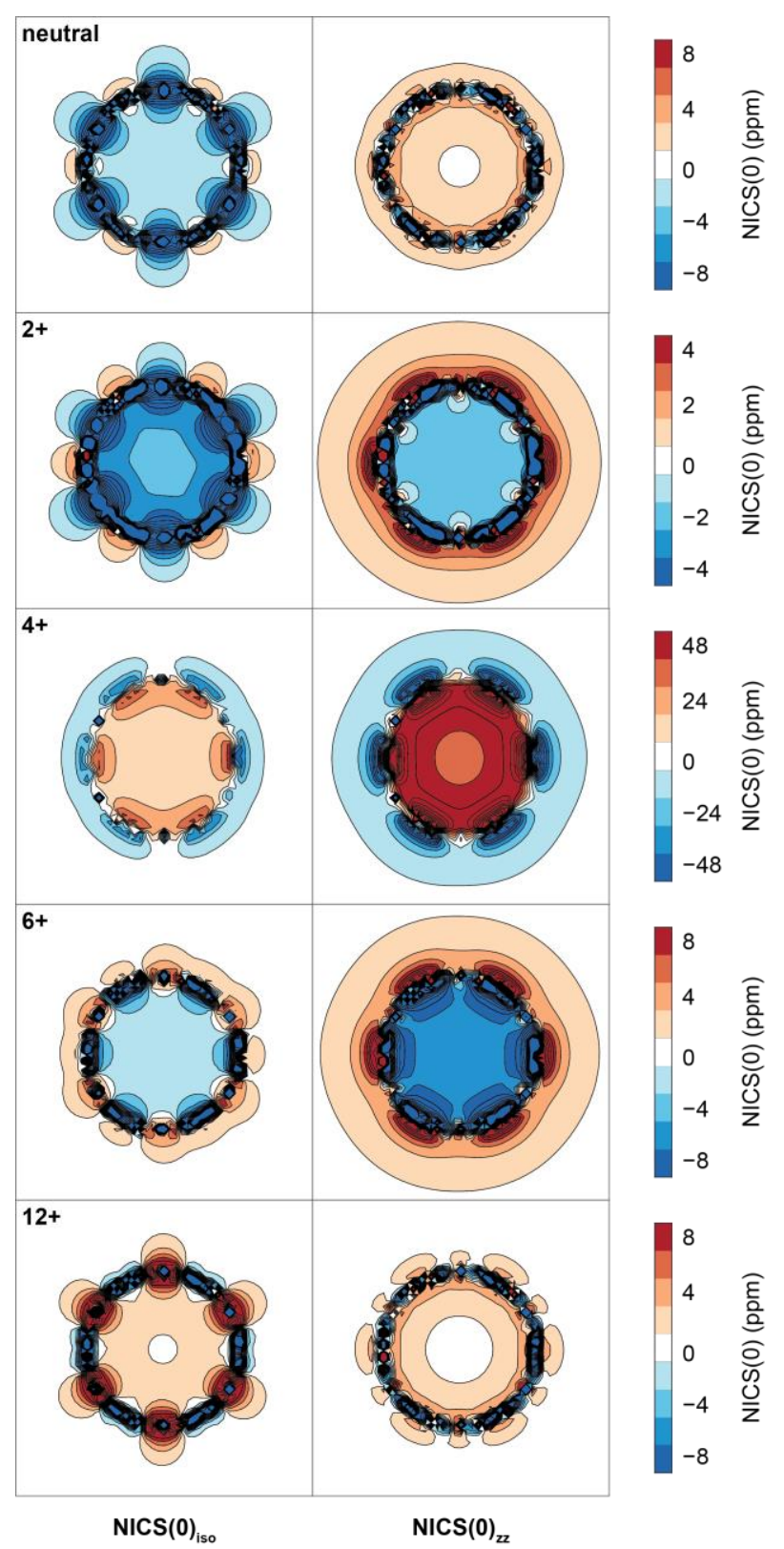

Figure 2. Plots of $\mathrm{NICS}(0)_{\text {iso }}$ and $\mathrm{NICS}(0)_{z z}$ for $c-\mathrm{P} 6\left[\mathrm{~b}_{6}\right]^{Q}$ for $Q=0,+2,+4,+6$ and +12 calculated using the BLYP35 functional. The calculation for $Q=+2$ assumes a symmetry-broken unrestricted singlet-state. 

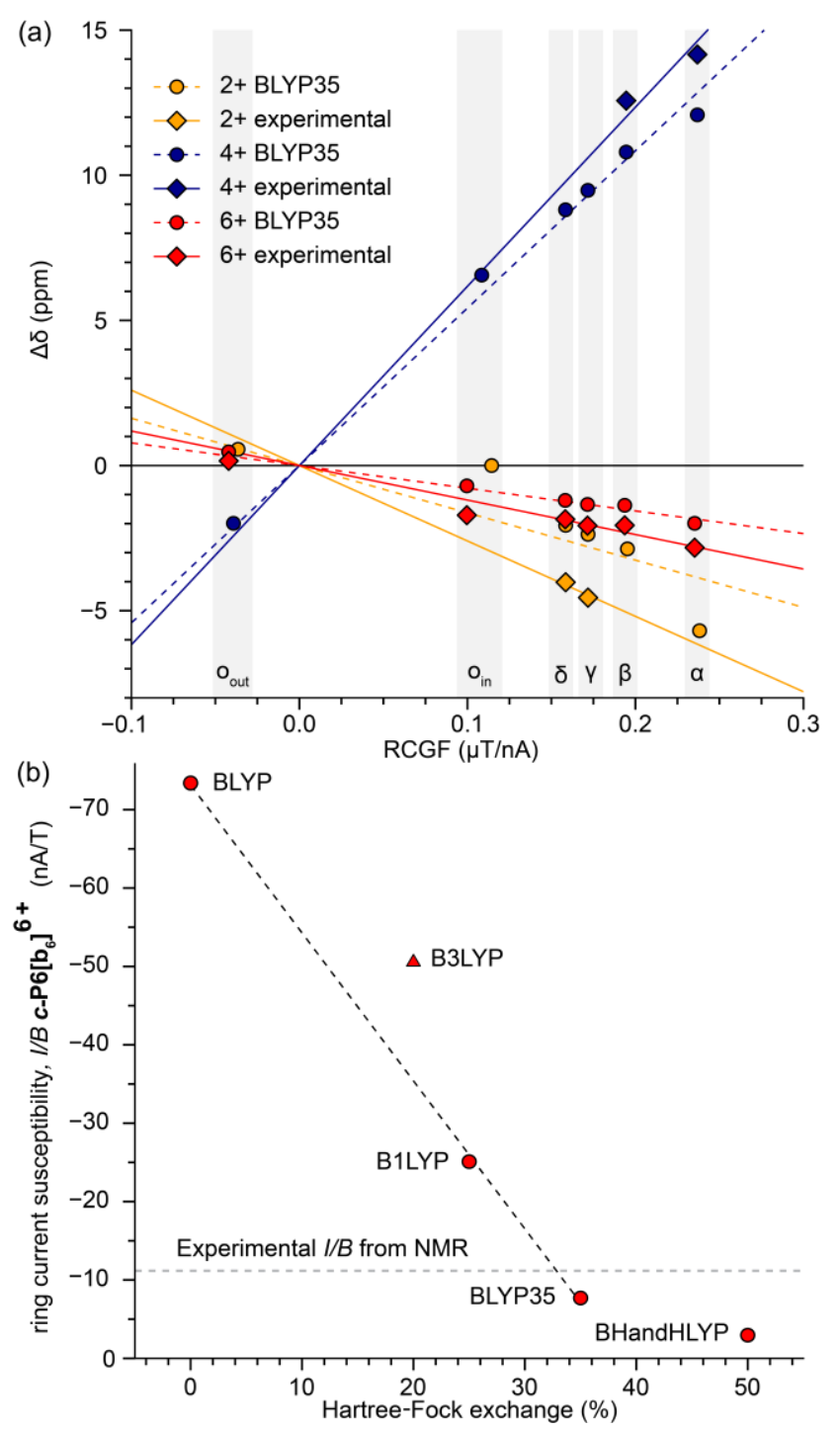

Figure 3. (a) Plot of $\Delta \delta$ vs. RCGF for $c-P 6\left[b_{6}\right]^{Q},(Q=+2,+4$ and +6$)$ showing experimental points and BLYP35 calculated points. (b) Plot of predicted ring current susceptibility $(I / B)$ against the fraction of Hartree-Fock exchange included in the DFA for $\boldsymbol{c}-\mathbf{P} 6\left[\mathbf{b}_{6}\right]^{6+}$.

The choice of DFA has a dramatic effect on the predicted aromaticity. Figure $3 \mathrm{~b}$ shows how the ring-current susceptibility of $\mathbf{c}-\mathbf{P} 6\left[\mathbf{b}_{6}\right] \cdot \mathbf{T 6}^{6+}$ varies with the proportion of Hartree-Fock exchange within the $\mathrm{B}(1) \mathrm{LYP}$ family of global (hybrid) DFAs. BLYP, which contains no HF exchange, predicts an extremely high ring current susceptibility. In contrast, BHandHLYP appears to suffer from over-localization relative to experiment, and calculates near-zero ring current susceptibility. The B3LYP functional is not a member of this series, but contains $20 \% \mathrm{HF}$ exchange and provides a high estimate of the ring-current susceptibility, consistent with Matito and coworkers' results. The experimental ring-current susceptibility is close to that from the DFA with $35 \% \mathrm{HF}$, and as we showed above this DFA reliably reproduces experimental NMR chemical shifts and ring current susceptibilities for all the measured oxidation states.

The changes in chemical shift for the other six-porphyrin nanorings, $c-\mathrm{P} 6\left[\mathrm{e}_{6}\right] \cdot \mathrm{T} 6^{\star Q}, \quad c-\mathrm{P} 6\left[\mathrm{be}_{5}\right] \cdot \mathrm{T} 6^{* Q}$ and $c-\mathrm{P} 6\left[\mathrm{~b}_{5} \mathrm{e}\right] \cdot \mathrm{T}{ }^{Q}$ also match well with the predictions from DFT calculations using
BLYP35 (see Table 2 and details in Supporting Information). There is a clear alternation between shielding $(\Delta \delta<0)$ of template protons when the Hückel $\pi$-electron count is $N_{e}=4 n+$ 2 , and deshielding $(\Delta \delta>0)$ when $N_{e}=4 n$. Adding or removing a $\mathrm{C} \equiv \mathrm{C}$ unit changes the electron count by 2 and changes the direction of the global ring current (diatropic vs. paratropic) in agreement with the predictions of the Hückel rule (Figure 4).

Table 2. Experimental (and BLYP35-calculated) changes in chemical shift (in ppm) and ring current susceptibilities $/ / B$ in six-porphyrin nanorings.

\begin{tabular}{|c|c|c|c|c|c|c|}
\hline species & $Q^{[a]}$ & $N_{e}^{[b]}$ & $\Delta \delta(0)$ & $\Delta \delta(\alpha)$ & $\Delta \delta(\beta)$ & $I / B(\mathrm{nA} / \mathrm{T})$ \\
\hline \multirow{3}{*}{$c-\mathrm{P} 6\left[\mathrm{e}_{6}\right] \cdot \mathrm{T} 6^{\star}$} & +2 & 70 & $\begin{array}{l}-5.92 \\
(-8.30)\end{array}$ & $\begin{array}{l}-16.0 \\
(-21.3)\end{array}$ & $\begin{array}{l}-11.0 \\
(-15.8)\end{array}$ & $\begin{array}{l}-55 \\
(-76)\end{array}$ \\
\hline & +4 & 68 & $(\overline{5.67})$ & $\begin{array}{l}27.3 \\
(8.13)\end{array}$ & $\begin{array}{c}22.7 \\
(7.02)\end{array}$ & $\begin{array}{l}111 \\
(35)\end{array}$ \\
\hline & +6 & 66 & $\begin{array}{c}-2.43 \\
(-1.03)\end{array}$ & $\begin{array}{l}-4.40 \\
(-2.13)\end{array}$ & $\begin{array}{c}-3.13 \\
(-1.89)\end{array}$ & $\begin{array}{c}-17 \\
(-8.9)\end{array}$ \\
\hline \multirow{3}{*}{$c-\mathrm{P} 6\left[\mathrm{be}_{5}\right] \cdot \mathrm{T}^{*}$} & +4 & 70 & $(-\overline{4.57})$ & $\begin{array}{c}-15.2 \\
(-11.3)\end{array}$ & $\begin{array}{c}-11.1 \\
(-8.72)\end{array}$ & $\begin{array}{l}-59 \\
(-43)\end{array}$ \\
\hline & & & & & & \\
\hline & +6 & 68 & $(\overline{1.06})$ & $\begin{array}{c}2.80 \\
(1.93)\end{array}$ & $\begin{array}{c}2.68 \\
(1.50)\end{array}$ & $\begin{array}{c}12 \\
(7.8)\end{array}$ \\
\hline \multirow{3}{*}{$c-\mathrm{P} 6\left[\mathrm{~b}_{5} \mathrm{e}\right] \cdot \mathrm{T} 6$} & +4 & 78 & $(-6.36)$ & $\begin{array}{c}-14.0 \\
(-14.0)\end{array}$ & $\begin{array}{c}-10.2 \\
(-10.4)\end{array}$ & $\begin{array}{l}-57 \\
(-54)\end{array}$ \\
\hline & & & & & & \\
\hline & +6 & 76 & $\overline{(0.61)}$ & $\begin{array}{c}2.17 \\
(1.16)\end{array}$ & $\begin{array}{c}1.92 \\
(1.20)\end{array}$ & $\begin{array}{c}9.5 \\
(5.3)\end{array}$ \\
\hline \multirow{3}{*}{$c-\mathrm{P} 6\left[\mathrm{~b}_{6}\right] \cdot \mathrm{T} 6$} & +2 & 82 & $(-\overline{0.56})$ & $(-5.69)$ & $(-2.87)$ & $\begin{array}{l}-26^{[c]} \\
(-16)\end{array}$ \\
\hline & +4 & 80 & $(\overline{8.56})$ & $\begin{array}{c}14.2 \\
(12.1)\end{array}$ & $\begin{array}{c}12.6 \\
(10.8)\end{array}$ & $\begin{array}{c}62 \\
(54)\end{array}$ \\
\hline & +6 & 78 & $\begin{array}{l}-1.87 \\
(-1.17)\end{array}$ & $\begin{array}{l}-2.83 \\
(-1.99)\end{array}$ & $\begin{array}{l}-2.06 \\
(-1.37)\end{array}$ & $\begin{array}{c}-12 \\
(-7.8)\end{array}$ \\
\hline
\end{tabular}

[a] Data for the neutral nanorings $(Q=0)$ are not shown here because they only exhibit local ring currents, but their experimental $\Delta \delta$ values are also well reproduced using BLYP35, see SI. [b] $N_{e}$ is the number of $\pi$-electrons in the Hückel circuit around the macrocycle. [c] The experimental $I / B$ for $\boldsymbol{c}$ $\mathbf{P} 6\left[\mathbf{b}_{6}\right] \cdot \mathbf{T 6}^{2+}$ comes from the measured $\Delta \delta(\gamma)$ and $\Delta \delta(\delta)$ values. 


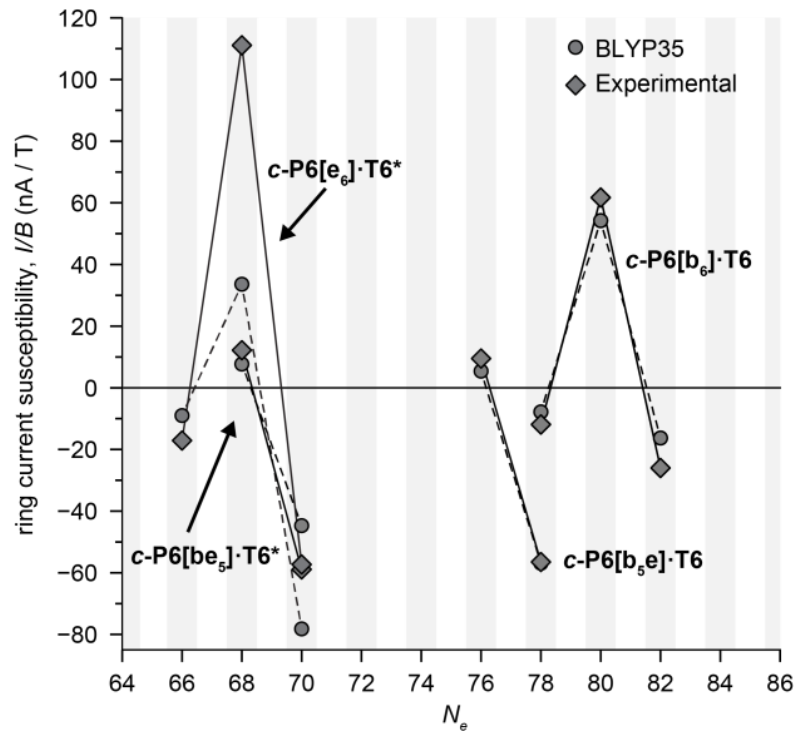

Figure 4. Relationship between ring current susceptibility $(/ / B)$ and $\pi$-electron count showing calculated points from BLYP35 and values derived from experimental NMR data for the full set of six-porphyrin nanorings from Figure 1 (b)-(e) in various oxidation states.

In conclusion, we have shown that the experimental NMR chemical shift changes, $\Delta \delta$, in six-porphyrin nanorings, in a range of oxidation states, are well reproduced by DFT calculations using hybrid exchange-correlation functionals such as BLYP35 and LC- $\omega$ hPBE $(\omega=0.1)$. The patterns of $\Delta \delta$, as a function of oxidation state and as a function of the number of linking $\mathrm{C} \equiv \mathrm{C}$ units, provide compelling evidence for the presence of global ring currents. Although the ring current susceptibilities of $c-\mathrm{P} 6\left[\mathrm{~b}_{6}\right] \cdot \mathbf{T 6}^{Q}$ predicted by BLYP35 are small compared with those from B3LYP, they are still larger than the value for benzene $(I / B \approx 11 \mathrm{nA} / \mathrm{T}),{ }^{[8,13,14]}$ and it would be wrong to say that these species show negligible global aromaticity.

\section{Acknowledgements}

We thank the ERC (Advanced Grants 320969 and 885606) and the EPSRC (grants EP/M016110, EP/R029229/1 and EP/N017188/1) for support. MDP thanks UNSW for a Scientia Fellowship. Computational services were provided by the National Computational Infrastructure ( $\mathrm{NCl})$, which is supported by the Australian Government, and the Advanced Research Computing Service at the University of Oxford. We also thank $\mathrm{Dr}$ Irene Casademont-Reig and Dr Eduard Matito for sharing their data with us.

Keywords: aromatic $\cdot$ macrocycle $\cdot$ density functional theory • porphyrin $\bullet$ ring current

[1] a) P. von R. Schleyer, H. Jiao, Pure \& Appl. Chem. 1996, 68, 209-218 b) T. M. Krygowski, M. K. Cyrañski, Z. Czarnocki, G. Häfelinger, A. R. Katritzky, Tetrahedron 2000, 56, 1783-1796; c) T. M. Krygowski, M. K. Cyrañski, Chem. Rev. 2001, 101, 1385-1419; d) M. Kertesz, C. H. Choi S. Yang, Chem. Rev. 2005, 105, 3448-3481; e) F. Feixas, E. Matito, J Poater, M. Solà, Chem. Soc. Rev. 2015, 44, 6434-6451; f) R Gershoni-Poranne, A. Stanger, Chem. Soc. Rev. 2015, 44, 6597-6615.
[2] M. D. Peeks, T. D. W. Claridge, H. L. Anderson, Nature 2017, 541, 200-203.

[3] M. D. Peeks, M. Jirasek, T. D. W. Claridge, H. L. Anderson, Angew. Chem., Int. Ed. 2019, 58, 15717-15720; Angew. Chem. 2019, 131 15864-15867.

[4] M. D. Peeks, J. Q. Gong, K. McLoughlin, T. Kobatake, R. Haver, L. M Herz, H. L. Anderson, J. Phys. Chem. Lett. 2019, 10, 2017-2022.

[5] M. Rickhaus, M. Jirasek, L. Tejerina, H. Gotfredsen, M. D. Peeks, R Haver, H.-W. Jiang, T. D. W. Claridge, H. L. Anderson, Nat. Chem. 2020, 12, 236-241.

[6] S. M. Kopp, H. Gotfredsen, J.-R. Deng, T. D. W. Claridge, H. L. Anderson, J. Am. Chem. Soc. 2020, 142, 19393-19401.

[7] M. Jirásek, M. Rickhaus, L. Tejerina, H. L. Anderson, J. Am. Chem Soc. 2021, 143, 2403-2412.

[8] M. Jirásek, H. L. Anderson, M. D. Peeks, Acc. Chem. Res. 2021, 54 3241-3252.

[9] I. Casademont-Reig, R. Guerrero-Avilés, E. Ramos-Cordoba, M Torrent-Sucarrat, E. Matito, Angew. Chem., Int. Ed. 2021, 60, 2408024088; Angew. Chem. 2021, 133, 24282-24290

[10] a) A. J. Cohen, P. Mori-Sánchez, W. Yang, Science 2008, 321 , 792-794; b) T. M. Henderson, A. F. Izmaylov, G. Scalmani, G. E. Scuseria, J. Chem. Phys. 2009, 131, 044108; c) M. Kaupp, M. Renz, M. Parthey, M. Stolte, F. Würthner, C. Lambert, Phys. Chem. Chem. Phys. 2011, 13, 16973-16986.

[11] a) M. Renz, K. Theilacker, C. Lambert, M. Kaupp, J. Am. Chem. Soc 2009, 131, 16292-16302; b) M. Parthey, M. Kaupp, Chem. Soc. Rev. 2014, 43, 5067-5088.

[12] N. Sylvetsky, A. Banerjee, M. Alonso, J. M. L. Martin, J. Chem. Theory Comput. 2020, 16, 3641-3653

[13] a) H. Fliegl, D. Sundholm, S. Taubert, J. Jusélius, W. Klopper, J. Phys Chem. A 2009, 113, 8668-8676; b) G. Monaco, R. Zanasi, J. Phys Chem. A 2014, 118, 1673-1683.

[14] a) D. Sundholm, H. Fliegl, R. J. F. Berger, WIREs Comput. Mol. Sci. 2016, 6, 639-678; b) H. Fliegl, S. Taubert, O. Lehtonen, D. Sundholm, Phys. Chem. Chem. Phys. 2011, 13, 20500-20518.

[15] R. C. Haddon, Tetrahedron 1972, 28, 3613-3633. 


\section{Entry for the Table of Contents}

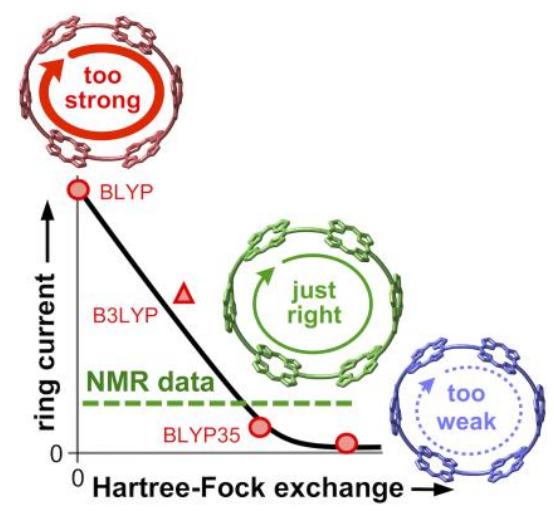

Insert text for Table of Contents here. To be or not to be like benzene? Fortunately porphyrin nanoring template complexes provide a wealth of experimental NMR spectroscopy data, which are sensitive to global ring currents and which can be used to guide computational work on answering this question. If the density functional approximation is chosen to reproduce the NMR data, the results confirm that nanoring cations are globally (anti)aromatic, consistent with Hückel's rule.

Institute and/or researcher Twitter usernames: @HLAGroupOx, @PeeksGroup, @Martinp23 